\title{
Accurate Calculations of Properties of the Two-Tube Electrostatic Lens. I. Improved Digital Methods for the Precise Calculation of Electric Fields and Trajectories
}

\author{
S. Natali,* D. Di Chio,* and C. E. Kuyatt \\ Institute for Basic Standards, National Bureau of Standards, Washington, D.C. 20234
}

(September 29, 1971)

\begin{abstract}
Digital methods of high precision have been developed for the calculation of electric fields and trajectories in electrostatic lenses and a computer program was written to apply these methods to the two-tube lens. The increased precision results from the use of nine-point formulas in the relaxation calculation of potentials in place of previously used five-point formulas and from the use of an improved predictor-corrector method for the calculation of trajectories. Trajectories obtained with these methods are sufficiently precise to determine third-order aberration coefficients.
\end{abstract}

Key words: Calculation of electric fields; calculation of electron trajectories; electron lens; 9-point relaxation formulas; predictor-corrector method; two-tube electrostatic lens.

\section{Introduction}

As part of a general program to determine precise properties of electrostatic lenses, highly precise digital methods were developed for the calculation of electric fields and trajectories in electrostatic lenses and a computer program was written to apply these methods to the two-tube lens. ${ }^{1}$ Our methods are easily generalizable to a wide class of electrostatic lenses.

The two-tube lens was chosen to test the digital methods since considerable data are available for this lens. Comparison of our results with the existing data shows that our methods have increased the accuracy of the calculation of lens properties and makes it possible, for the first time, to determine all of the thirdorder aberration coefficients of the two-tube lens for meridional trajectories.

\section{Calculation of the Potentials}

\subsection{Statement of the Problem}

Since a two-tube lens is cylindrically symmetric, the potential distribution inside the cylinders can be obtained by solving Laplace's equation in cylindrical coordinates (see fig. 1)

$$
\frac{\partial^{2} V(r, z)}{\partial r^{2}}+\frac{1}{r} \frac{\partial V(r, z)}{\partial r}+\frac{\partial^{2} V(r, z)}{\partial z^{2}}=0
$$

\footnotetext{
* Present address: Istituto di Fisica, Universita di Bari, Bari, Italy.

A detailed description of the computer program will be published separately.
}

giving as boundary conditions the potentials on a contour starting and ending on the axis. Let $V_{1}$ and $V_{2}$ be the potentials on the two cylinders, $D$ the inside diameter, and $S$ the size of the gap between the two cylinders. The following boundary conditions were used : (see fig. 1)

$$
\begin{array}{ll}
A B: & V=V_{1} \\
B C: & V=V_{1} \\
C D: & V=V_{1} \\
D E: & V=\text { linear variation from } V_{1} \text { to } \frac{1}{2}\left(V_{1}+V_{2}\right) \\
E F: & V=\frac{1}{2}\left(V_{1}+V_{2}\right)
\end{array}
$$

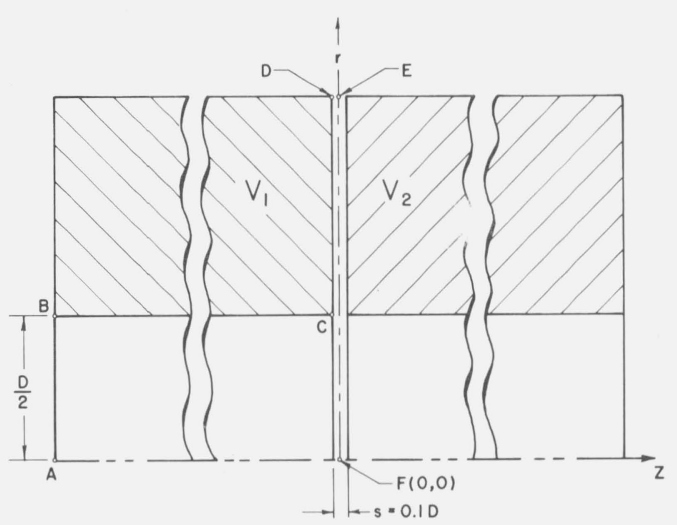

FigurE 1. Schematic of the two-tube electrostatic lens, showing $\mathrm{r}, \mathrm{z}$, coordinate system and boundary used in the relaxation calculation of potentials.

$$
A F=B C=3.5 D . \quad C D=1.25 D .
$$


In the actual calculations the distance $A F$ is typically $3.5 \mathrm{D}$. It is sufficient to calculate the potentials only inside $A B C D E F A$ since cylindrical symmetry requires

$$
V(-r, z)=V(r, z)
$$

and the symmetry about the midplane of the lens requires

$$
V(r,-z)=V_{1}+V_{2}-V(r, z) .
$$

In addition, once the solution $\mathrm{V}_{0}(r, z)$ is obtained for the case $V_{1}=0, V_{2}=1$, then the solution for any other potentials $V_{1}, V_{2}$ on the tubes can be obtained from the formula

$$
V(r, z)=V_{1}+\left(V_{2}-V_{1}\right) V_{0}(r, z)
$$

\subsection{Method of Solution}

Laplace's equation (eq 1) was solved by the relaxation method on a matrix of equally spaced points [1].2 The domain $A B C D E F A$ was divided into a network of equal sides $h$. Let $i$ be the index running along the $z$ axis, and $j$ the index along $r$. Let $V_{i, j}$ be the potential at mesh point $(i, j)$. Then the first order difference form of eq (1) is:

$$
\begin{array}{r}
\frac{V_{i, j+1}-2 V_{i, j}+V_{i, j-1}}{h^{2}}+\frac{V_{i, j+1}-V_{i, j-1}}{2 h r_{j}} \\
+\frac{V_{i+1, j}-2 V_{i, j}+V_{i-1, j}}{h^{2}}=0
\end{array}
$$

From eq (2) the relationship between $V_{i, j}$ and the four adjacent points is easily obtained:

${ }^{2}$ Figures in brackets indicate the literature references at the end of this paper.

$$
\begin{array}{r}
V_{i, j}=\frac{V_{i, j+1}+V_{i, j-1}+V_{i+1, j}+V_{i-1, j}}{4} \\
+\frac{1}{8 r_{j}}\left(V_{i, j+1}-V_{i, j-1}\right)
\end{array}
$$

Here we assume that $r_{j}$ is measured in units of $h$. On the axis where

$$
\lim _{r \rightarrow 0} \frac{1}{r} \frac{\partial V}{\partial r}=\left(\frac{\partial^{2} V}{\partial r^{2}}\right)_{r=0}
$$

eq (3) becomes

$$
V_{i, j}=\frac{1}{6}\left(4 V_{i, j+1}+V_{i+1, j}+V_{i-1, j}\right) .
$$

In order to increase the accuracy of the relaxation method, we have expressed $V_{i, j}$ in terms of the potentials of eight adjacent points. This is accomplished by using Stirling differentiation formulas [2] for five points at equal intervals in both the $r$ and $z$ directions. The resulting formulas, including those special formulas required for points near the boundaries, are listed in table 1.

The basic nine-point formula used to relax the network is Il, and its specialization to axial points is I2. Formula I3 was obtained from Il assuming symmetry about the axis. Formulas I4 and I6 which were used for points near the left boundary are just special cases of the five-point formulas given in eqs (3) and (5). Formula I5 was obtained from If and the relation

$$
V_{M+1, j}=2 V_{M, j}-V_{M-1, j} .
$$

TABLE 1. Formulas used for relaxation

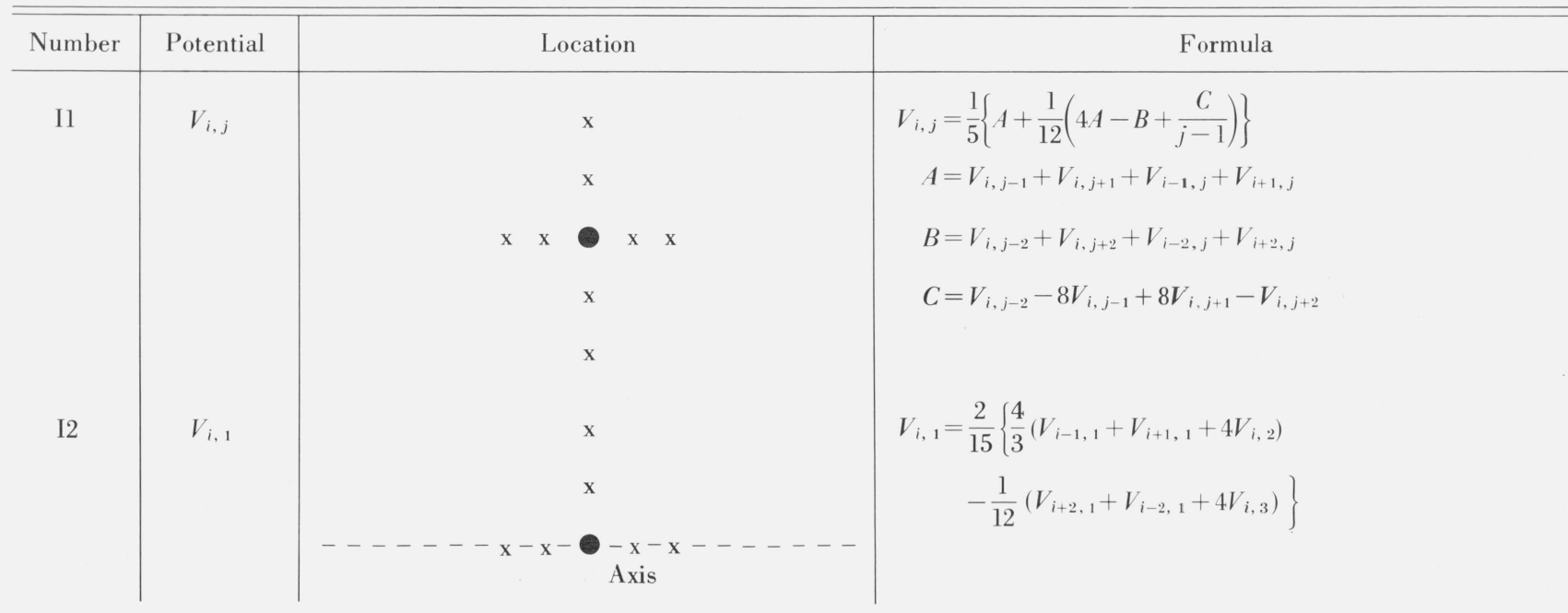


TABLE 1. Formulas used for relaxation-Continued

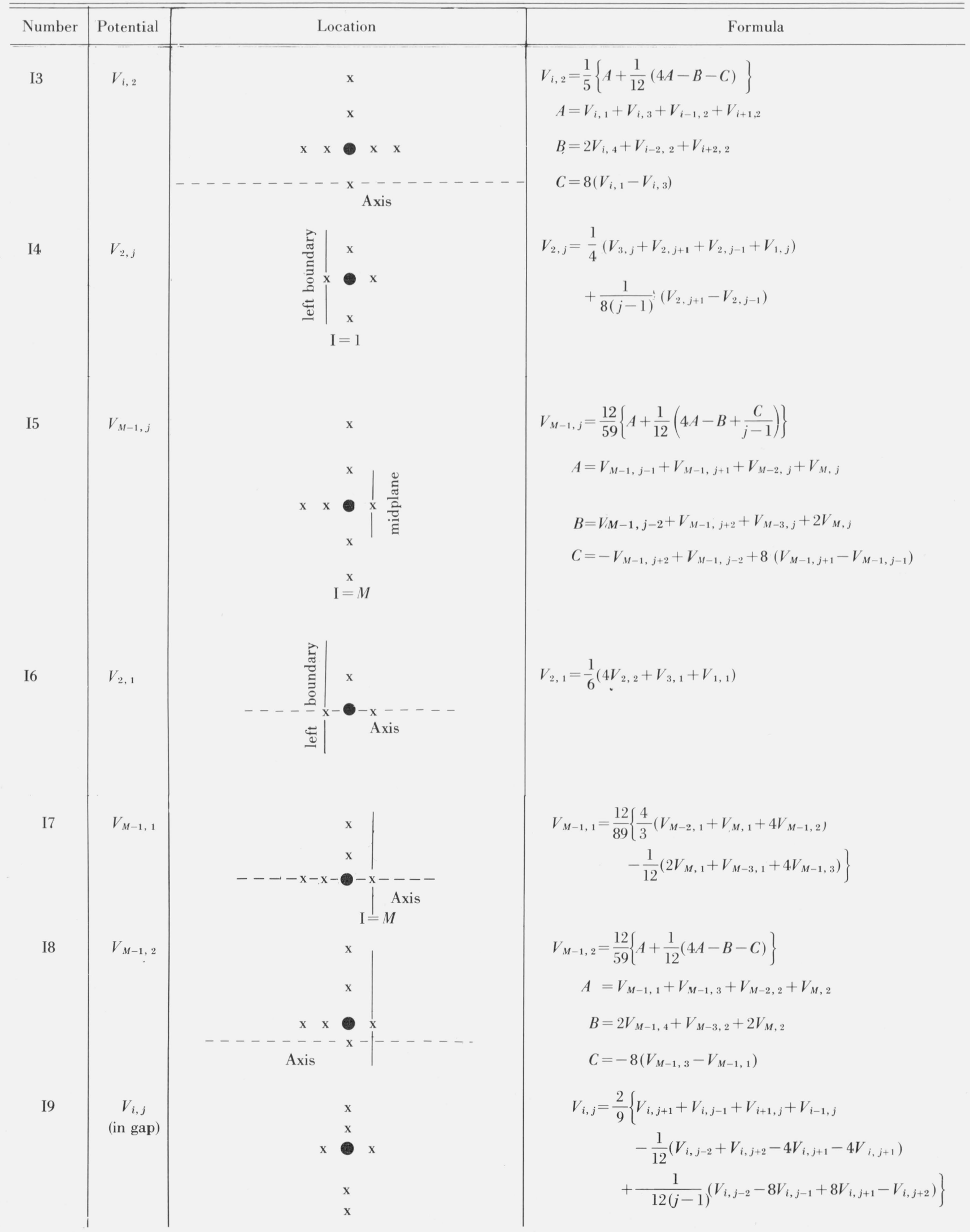


TABLE 1. Formulas used for relaxation-Continued

\begin{tabular}{|c|c|c|c|}
\hline Number & Potential & Location & Formula \\
\hline I10 & $V_{i, N-1}$ & $\begin{array}{c}\frac{\text { tube }}{\mathrm{x}} \mathrm{x}-\mathrm{x} \times \mathrm{x} \\
\mathrm{x}\end{array}$ & $\begin{aligned} V_{i, N-1} & =\frac{2}{9}\left(A-\frac{B}{12}+\frac{C}{2(N-2)}\right) \\
A & \left.=V_{i, N}+V_{i, N-2}+V_{i-\mid 1, N-1}+V_{i+1, N-1}\right) \\
B & =V_{i-2, N-1}+V_{i+2, N-1}-4\left(V_{i-1, N-1}+V_{i+1, N-1}\right) \\
C & =V_{i, N}-V_{i, N-2}\end{aligned}$ \\
\hline
\end{tabular}

Formula I7 was obtained from I2 and eq (6), and I8 was obtained from I3 and eq (6). Formula I9 was derived by substituting into Laplace's equation the three-point formula for the derivative with respect to $z$ and the five-point formulas for the derivatives with respect to $r$. Finally, Formula $\mathrm{Il} 0$ was derived by substituting into Laplace's equation the threepoint formulas for the derivatives with respect to $r$ and the five-point formula for the derivative with respect to $z$.

\subsection{Overrelaxation Procedure}

The relaxation method is based on successive iterations of formulas Il through I10 until the values assumed by the $V_{i, j}$ in two successive iterations differ by less than a preselected amount. Since the convergence is very slow, overrelaxation is used. The potential at point $(i, j)$ after the $m$ th iteration is then given by

$$
V_{i, j}^{m}=V_{i, j}^{m-1}+\omega\left(V_{i, j}^{* m}-V_{i, j}^{m-1}\right)
$$

where $V^{*}$ is the potential calculated from formulas Il through I10. The quantity $\omega$ is called the overrelaxation or acceleration factor, and its optimum value is problem dependent.

To determine $\omega$ we use the method of Carré [3]. Twelve iterations are first performed using a value $\omega=1.375$ which is certainly smaller than the optimal value $\omega_{0}$, and a quantity $W$ is calculated from the last three iterations:

$$
W=\frac{\sum_{n=1}^{N}\left|V_{n}^{(m+1)}-V_{n}^{(m)}\right|}{\sum_{n=1}^{N}\left|V_{n}^{(m)}-V_{n}^{(m-1)}\right|} .
$$

The next estimate of the acceleration factor is then calculated from

$$
\omega_{l}=\frac{2}{1+\sqrt{1-\frac{(W+\omega-1)^{2}}{W \omega^{2}}}}
$$

Carré has shown the importance of not exceeding the optimum value $\omega_{0}$, and finds empirically that a value

$$
\omega_{m}=\omega_{l}-\frac{1}{4}\left(2-\omega_{l}\right)
$$

is close to $\omega_{0}$ but never exceeds it. The next twelve iterations are then performed using the acceleration factor $\omega_{m}$ and new estimates of $W, \omega_{l}$, and $\omega_{m}$ ealculated from eqs $(8-10)$. The entire process is repeated twelve times or until two successive estimates $\omega_{l}$, $\omega_{l+1}$ from eq (9) satisfy the inequality

$$
\frac{\omega_{l+1}-\omega_{l}}{2-\omega_{l}}<0.05
$$

Succeeding iterations are then made with the last estimate of $\omega_{0}$ from eq (9) and are repeated until

$$
\left|V_{i, j}^{m}-V_{i, j}^{m-1}\right| \cdot \frac{W}{1-W} \leqslant \epsilon
$$

for all points on the mesh. The quantity $\epsilon$ is the desired precision for the potentials.

\subsection{Program Organization}

The radius of the lens was divided into 40 mesh points, with 320 mesh points along the axis for the half-lens. Hence, the program must relax 12,800 points. In addition, a $5 \times 100$ matrix is relaxed in the gap between the tubes, with 15 points overlapping the main matrix (see fig. 2). The time required to relax the complete network to a precision of $\epsilon=10^{-8}$ was about

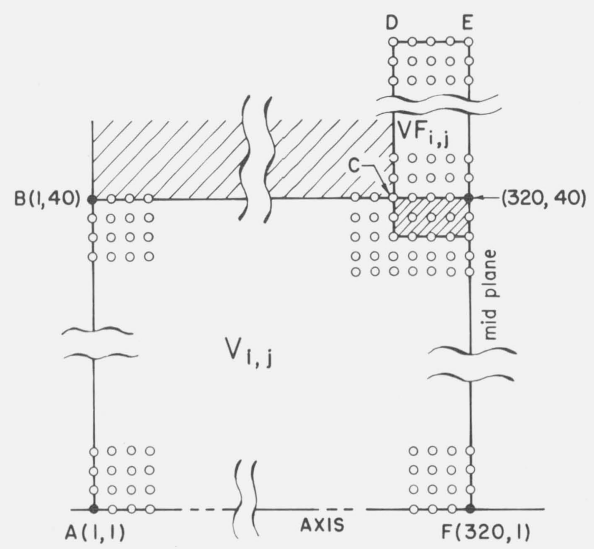

FIGURE 2. Layout of mesh points showing overlapping of the main matrix with the auxiliary matrix used in the lens gap. Crosshatched region is area of overlap. 
TABLE 2. Axial potentials $\mathrm{V}(\mathrm{z})$ for the two-tube electrostatic lens

$z=0$ at the center of the gap. $V_{1}=0, V_{2}=1$.

\begin{tabular}{|c|c|c|c|c|c|c|c|}
\hline \multirow{3}{*}{$Z / D$} & \multicolumn{7}{|c|}{$V(z)$} \\
\hline & \multicolumn{3}{|c|}{ gap $=0.1 \mathrm{D}$} & \multicolumn{4}{|c|}{ gap $=0$} \\
\hline & $\begin{array}{l}\text { Present } \\
\text { results }\end{array}$ & $\begin{array}{l}\text { Read } \\
\text { et al. }\end{array}$ & $\begin{array}{c}\text { Grivet- } \\
\text { Bernard }\end{array}$ & Verster & $\begin{array}{l}\text { Read } \\
\text { et al. }\end{array}$ & El-Kareh & $\begin{array}{l}\text { Grivet- } \\
\text { Bernard }\end{array}$ \\
\hline 0 & .500000 & .500000 & .500000 & .500000 & .500000 & .500000 & .500000 \\
\hline .025 & .467115 & .467206 & .467237 & .466896 & .466896 & & .467089 \\
\hline .05 & .434532 & .434703 & .434753 & .434101 & .434101 & & .434462 \\
\hline .075 & .402538 & .402789 & .402816 & .401911 & .401911 & & .402393 \\
\hline .1 & .371399 & .371715 & .371679 & .370599 & .370599 & .370606 & .371138 \\
\hline .125 & .341351 & .341722 & .341566 & .340406 & .340406 & & .340925 \\
\hline .15 & .312592 & .313005 & .312673 & .311533 & .311533 & & .311952 \\
\hline .175 & .285279 & .285722 & .285159 & .284138 & .284138 & & .284379 \\
\hline .2 & .259528 & .259991 & .259146 & .258334 & .258334 & .258327 & .258327 \\
\hline .225 & .235413 & .235884 & .234720 & .234195 & .234195 & & .233883 \\
\hline .25 & .212969 & .213438 & .211932 & .211752 & .211752 & & .211093 \\
\hline .3 & .173079 & .173517 & .171309 & .171921 & .171921 & .171925 & .170510 \\
\hline .35 & .139566 & .139957 & .137100 & .138518 & .138518 & & .136380 \\
\hline .4 & .111851 & .112180 & .108812 & .110938 & .110938 & .110939 & .108190 \\
\hline .45 & .089212 & .089479 & .085771 & .088438 & .088438 & & .085253 \\
\hline .5 & .070897 & .071103 & .067235 & .070253 & .070253 & .070247 & .066813 \\
\hline .6 & .044436 & .044536 & .040802 & .044009 & .044009 & .044012 & .040542 \\
\hline .7 & .027676 & .027690 & .024483 & .027403 & .027403 & .027405 & .024331 \\
\hline .8 & .017178 & .017141 & .014590 & .017006 & .017006 & .017003 & .014504 \\
\hline .9 & .010642 & .010571 & .008658 & .010535 & .010535 & .010538 & .008611 \\
\hline 1.0 & .006586 & .006544 & .005125 & .006520 & .006520 & .006520 & .005100 \\
\hline 1.1 & .004074 & .004048 & .003029 & .004033 & .004033 & .004029 & .003016 \\
\hline 1.2 & .002519 & .002501 & .001789 & .002494 & .002494 & .002498 & .001782 \\
\hline 1.3 & .001557 & .001545 & .001056 & .001542 & .001542 & .001543 & .001053 \\
\hline 1.4 & .000962 & .000954 & .000623 & .000953 & .000953 & .000951 & .000621 \\
\hline 1.5 & .000595 & .000589 & .000368 & .000589 & .000589 & .000591 & .000367 \\
\hline 1.6 & .000368 & .000364 & .000217 & .000364 & .000364 & & .000217 \\
\hline 1.7 & .000227 & .000225 & .000128 & .000225 & .000225 & & .000128 \\
\hline 1.8 & .000141 & .000139 & .000075 & .000139 & .000139 & & .000075 \\
\hline 1.9 & .000087 & .000086 & .000044 & .000086 & .000086 & & .000045 \\
\hline 2.0 & .000054 & .000053 & .000026 & .000053 & .000053 & & .000026 \\
\hline 2.1 & .000033 & .000033 & .000015 & .000033 & .000033 & & .000016 \\
\hline 2.2 & .000020 & .000020 & .000009 & .000020 & .000020 & & .000009 \\
\hline 2.3 & .000013 & .000013 & .000005 & .000013 & .000013 & & .000005 \\
\hline 2.4 & .000008 & .000008 & .000003 & .000008 & .000008 & & .000003 \\
\hline 2.5 & .000005 & .000005 & .000002 & .000005 & .000005 & & .000002 \\
\hline
\end{tabular}

16 min on an IBM 360/65 computer, using double precision arithmetic $(\sim 15$ decimal digits $)$.

\subsection{Results}

In table 2 the potentials we obtain along the axis are compared with potentials obtained with other methods. Our potentials are in agreement with Verster's calculated potentials [4] to within 0.3 percent. The systematic difference between our results and those of Verster is probably due to a difference in gap size, which in the present calculations is $0.1 D$ while Verster uses a zero gap.

Also given are potentials calculated with the Grivet-
Bernard approximation [6] for gap widths of $0.1 \mathrm{D}$ and zero. The agreement with our results and also with the results of Verster is good close to the gap but becomes progressively poorer away from the gap as could be expected with the approximations used by Grivet and Bernard. Note that the systematic difference between our results and those of Verster is in the same direction and roughly the same size as the differences between the Grivet-Bernard potentials for the same gap sizes.

We have also included the results of a calculation for the zero-gap case carried out by El-Kareh [5]. The agreement with Verster's calculation is extremely good. It should be noted that in deriving lens properties, 
El-Kareh uses the Grivet-Bernard approximation for the axial potentials.

The Grivet-Bernard approximation assumes a linear potential variation across the gap, while our method of continuing the relaxation process into the gap shows that the actual potential variation in the gap differs substantially from linear, as shown in table 3 . To assess the effect of the nonlinear potential variation in the gap, we made special calculations assuming a linear variation in the gap. It was found that the change in axial potential and lens properties was less than 0.1 percent.

TABLE 3. Linearly interpolated potentials compared to values from relaxation in the lens gap

$V_{1}=1, V_{2}=20 . l=$ distance measured from edge of tube along inner diameter of the tube. $s=$ gap width $=0.1 \mathrm{D}$

\begin{tabular}{l|c|c}
\hline \hline \multicolumn{1}{c|}{$l / s$} & Interpolated & Relaxation \\
\hline 0 & 1.000 & 1.000 \\
.125 & 3.375 & 3.987 \\
.25 & 5.750 & 6.315 \\
.375 & 8.125 & 8.441 \\
.5 & 10.500 & 10.500 \\
.625 & 12.875 & 12.559 \\
.75 & 15.250 & 14.685 \\
.875 & 17.725 & 17.013 \\
1.0 & 20.000 & 20.000 \\
\hline
\end{tabular}

Finally, very recent calculations of Read et al. [7] are given. For the zero-gap case, the same infinite series was evaluated by Read et al., as by Verster and by El-Kareh. The agreement is extremely good. For the finite-gap case, Read et al. use a new method involving a determination of charge distributions on the tubes which give agreement with the boundary conditions. The agreement with our results is within 0.0005 .

\section{The Ray Tracing Program}

\subsection{Statement of the Problem}

Using the potentials obtained by relaxation we must solve numerically the Lorentz equation:

$$
\mathbf{F}=-e \mathbf{E}
$$

where $\mathbf{F}$ is the force on the electron, and $\mathbf{E}$ is the electric field which must be calculated from the potentials at the mesh points. We restrict to the nonrelativistic case and to cylindrically symmetric electrostatic fields. In addition we consider only meridional rays, that is, rays lying in a plane through the axis of the lens. Equation (13) then reduces to the two equations:

$$
\left.\begin{array}{l}
\frac{d^{2} z}{d t^{2}}=-\frac{e}{m} E_{z}(r, z) \\
\frac{d^{2} r}{d t^{2}}=-\frac{e}{m} E_{r}(r, z) .
\end{array}\right\}
$$

Making the change of variables

$$
\left.\begin{array}{rl}
\tau^{2} & =\frac{2 e}{m} t^{2} \\
u & =\frac{d z}{d \tau} \\
v & =\frac{d r}{d \tau}
\end{array}\right\}
$$

eqs (15) become

$$
\left.\begin{array}{l}
\frac{d z}{d \tau}=u(r, z) \\
\frac{d u}{d \tau}=-\frac{1}{2} E_{z}(r, z) \\
\frac{d r}{d \tau}=v(z, r) \\
\frac{d v}{d \tau}=-\frac{1}{2} E_{r}(r, z) .
\end{array}\right\}
$$

We express the initial conditions as

$$
\left.\begin{array}{l}
z\left(\tau_{0}\right)=z_{0} \\
\left(\frac{d z}{d \tau}\right)_{0}=u_{0} \\
r\left(\tau_{0}\right)=r_{0} \\
\left(\frac{d z}{d \tau}\right)_{0}=v_{0} .
\end{array}\right\}
$$

\subsection{Method of Numerical Solution}

The numerical solution of the system of eqs (16) is of particular difficulty when a high precision is required. After a number of attempts using previous methods [1] we have chosen to use the predictorcorrector method of Hamming [8] where error and stability can be checked point by point. The important property of the classical predictor-corrector method is that it can be iterated until the required precision in the solution is reached. Hamming's method has the additional advantage of setting limits on the error which is accumulated along the integration. The method is stable in the sense that the difference between the numerical solution and the true solution decreases as the number of points of integration increases.

The predictor-corrector method seems therefore ideal for the calculation of trajectories. It has, however, two disadvantages: (1) It is not "self-starting"; hence, several starting points must be calculated with a different method. (2) It is desirable that at least several points be calculated in each mesh. Since the points are calculated at equal time intervals $h$ it may happen 
that when the electron accelerates the stability criteria require the interval $h$ to be reduced by a factor of 2 . Then the predictor-corrector method needs, to proceed, points calculated at intervals $\mathrm{h} / 2$, half of which do not exist. The trajectory must therefore be reinitiated with a different method.

Whenever required, the trajectory is reinitialized using the Runge-Kutta method. Reinitialization is only required when the electron moves toward the higher potential part of the lens. The effect of the occasional use of the Runge-Kutta method was checked by using the reversibility of electron trajectories. Several trajectories were calculated in both directions and found to coincide to within 0.01 percent.

A negligible error is expected when the trajectory is begun, this process being always performed in a zero-field region.

\subsection{Trajectory Initiation by the Runge-Kutta Method}

The numerical solution of eqs (16) uses the Rungenutta method to calculate the first seven points of the trajectory. Continuation of the solution then proceeds with the predictor-corrector method. Whenever he stability conditions fail to be satisfied at some point along the trajectory, the Runge-Kutta method is again applied to reinitialize the trajectory before continuing with the predictor-corrector method.

The Runge-Kutta formulas utilized are as follows:

$$
\left.\begin{array}{l}
z_{i+1}=z_{i}+\frac{1}{6}\left(k_{0}+2 k_{1}+2 k_{2}+k_{3}\right) \\
u_{i+1}=u_{i}+\frac{1}{6}\left(m_{0}+2 m_{1}+2 m_{2}+m_{3}\right) \\
r_{i+1}=r_{i}+\frac{1}{6}\left(n_{0}+2 n_{1}+2 n_{2}+n_{3}\right)
\end{array}\right\}
$$

where

$$
\begin{aligned}
k_{0} & =h u_{i} \\
n_{0} & =h v_{i} \\
m_{0} & =h E_{z}\left(r_{i}, z_{i}\right) \\
p_{0} & =h E_{r}\left(r_{i}, z_{i}\right) \\
k_{1} & =h \cdot\left(u_{i}+\frac{1}{2} m_{0}\right) \\
n_{1} & =h \cdot\left(v_{i}+\frac{1}{2} p_{0}\right) \\
m_{1} & =h \cdot E_{z}\left(r_{i}+\frac{n_{0}}{2}, z_{i}+\frac{k_{0}}{2}\right) \\
p_{1} & =h \cdot E_{r}\left(r_{i}+\frac{n_{0}}{2}, z_{i}+\frac{k_{0}}{2}\right) \\
k_{2} & =h \cdot\left(u_{i}+\frac{1}{2} m_{1}\right)
\end{aligned}
$$

$$
\begin{aligned}
n_{2} & =h \cdot\left(v_{i}+\frac{1}{2} p_{1}\right) \\
m_{2} & =h \cdot E_{z}\left(r_{i}+\frac{n_{1}}{2}, z_{i}+\frac{k_{1}}{2}\right) \\
p_{2} & =h \cdot E_{r}\left(r_{i}+\frac{n_{1}}{2}, z_{i}+\frac{k_{1}}{2}\right) \\
k_{3} & =h \cdot\left(u_{i}+m_{2}\right) \\
n_{3} & =h \cdot\left(v_{i}+p_{2}\right) \\
m_{3} & =h \cdot E_{z}\left(r_{i}+n_{2}, z_{i}+k_{2}\right) \\
p_{3} & =h \cdot E_{r}\left(r_{i}+n_{2}, z_{i}+k_{2}\right)
\end{aligned}
$$

with

$$
\begin{aligned}
& E_{z}=-E_{z} / 2 \\
& E_{r}=-E_{r} / 2
\end{aligned}
$$

$E_{z}$ and $E_{r}$ are obtained by differentiating with respect to $r$ and $z$ the Lagrange interpolating polynomial used to determine the potential at a given point from the values of the potentials at surrounding mesh points (see secs. 2.5 and 2.6) .

The time interval $h=\Delta \tau$ at the beginning is taken equal to $1 /\left(n \sqrt{V_{\max }}\right)$, thus assuring that the trajectory will be calculated for at least $n$ points per mesh. Then $h$ is reduced, if necessary, to satisfy the Hamming stability condition:

$$
\begin{gathered}
h k<0.4 \\
k=\left|\frac{\partial u}{\partial z}\right|+\left|\frac{\partial v}{\partial r}\right|+\left|\frac{\partial E_{z}}{\partial u}\right|+\left|\frac{\partial E_{r}}{\partial v}\right|
\end{gathered}
$$

In this way, after the seventh point is calculated from the Runge-Kutta method, the predictor-corrector method is started with points spaced by an $h$ which satisfies the stability condition.

\subsection{Trajectory Continuation by Hamming "Predictor- Corrector" Method}

The predictor-corrector formulas, as used in our program, are as follows:

\section{Predictor}

$$
\begin{aligned}
& z_{i+1}^{(0)}=z_{i-3}+\frac{4}{3} h\left[2 u_{i}-u_{i-1}+2 u_{i-2}\right] \\
& \begin{array}{r}
u_{i+1}^{(0)}=u_{i-3}+\frac{4}{3} h\left[2 E_{z}\left(r_{i}, z_{i}\right)-E_{z}\left(r_{i-1}, z_{i-1}\right)\right. \\
\left.+2 E_{z}\left(z_{i-2}, r_{i-2}\right)\right] \\
r_{i+1}^{(0)}=r_{i-3}+\frac{4}{3} h\left[2 v_{i}-v_{i-1}+2 v_{i-2}\right]
\end{array} \\
& \begin{array}{r}
v_{i+1}^{(o)}=v_{i-3}+\frac{4}{3} h\left[2 E_{z}\left(r_{i}, z_{i}\right)-E_{z}\left(r_{i-1}, z_{i-1}\right)\right. \\
+
\end{array}
\end{aligned}
$$


Modifier

$$
\begin{aligned}
& \bar{z}_{i+1}^{(0)}=z_{i+1}^{(0)}+\frac{112}{121}\left(z_{i}-z_{i}^{(0)}\right) \\
& \bar{u}_{i+1}^{(0)}=u_{i+1}^{(0)}+\frac{112}{121}\left(u_{i}-u_{i}^{(0)}\right) \\
& \bar{r}_{i+1}^{(0)}=r_{i+1}^{(0)}+\frac{112}{121}\left(r_{i}-r_{i}^{(0)}\right) \\
& \bar{v}_{i+1}^{(0)}=v_{i+1}^{(0)}+\frac{112}{121}\left(v_{i}-v_{i}^{(0)}\right)
\end{aligned}
$$

\section{Corrector}

$$
\begin{aligned}
z_{i+1}^{(j+1)}= & \frac{1}{8}\left[9 z_{i}-z_{i-2}+3 h\left(u_{i+1}^{(j)}+2 u_{i}-u_{i-1}\right)\right] \\
u_{i+1}^{(j+1)}= & \frac{1}{8}\left\{9 u_{i}-u_{i-2}+3 h\left[\left(E_{z}^{(j)}\right)_{i+1}+2\left(E_{z}\right)_{i}\right.\right. \\
& \left.\left.-\left(E_{z}\right)_{i-1}\right]\right\} \\
r_{i+1}^{(j+1)}= & \frac{1}{8}\left[9 r_{i}-r_{i-2}+3 h\left(v_{i+1}^{(j)}+2 v_{i}-v_{i-1}\right)\right] \\
v_{i+1}^{(j+1)}= & \frac{1}{8}\left\{9 v_{i}-v_{i-2}+3 h\left[\left(E_{r}^{(j)}\right)_{i+1}+2\left(E_{r}\right)_{i}\right.\right. \\
& \left.\left.-\left(E_{r}\right)_{i-1}\right]\right\}
\end{aligned}
$$

Ultimate Value [superscript $f$ indicates the final values from eqs (22)]

$$
\begin{aligned}
& z_{i+1}=z_{i+1}^{(f)}+\frac{9}{121}\left(z_{i+1}^{(0)}-z_{i+1}^{(f)}\right) \\
& u_{i+1}=u_{i+1}^{(f)}+\frac{9}{121}\left(u_{i+1}^{(0)}-u_{i+1}^{(f)}\right) \\
& r_{i+1}=r_{i+1}^{(f)}+\frac{9}{121}\left(r_{i+1}^{(0)}-r_{i+1}^{(f)}\right) \\
& v_{i+1}=v_{i+1}^{(f)}+\frac{9}{121}\left(v_{i+1}^{(0)}-v_{i+1}^{(f)}\right)
\end{aligned}
$$

The corrector formulae are iterated until the required precision in $z, r, u$, and $v$ is reached, that is, until the inequalities (24) are satisfied:

$$
\begin{aligned}
& \frac{h^{2} b_{-1}^{2} k_{1} k_{1}^{\prime}}{\left(1-h b_{-1} k_{1}^{\prime}\right)}<\mathrm{C}_{1} \\
& \frac{h^{2} b_{-1}^{2} k_{2} k_{2}^{\prime}}{\left(1-h b_{-1} k_{2}^{\prime}\right)}<\mathrm{C}_{2}
\end{aligned}
$$

where

$$
\begin{aligned}
b_{-1} & =\frac{3}{8} \\
k_{1} & =\left|u^{(j)}-u^{(j-1)}\right|_{i+1}+\left|v^{(j)}-v^{(j-1)}\right|_{i+1} \\
k_{1}^{\prime} & =\left|\frac{\partial u}{\partial z}\right|_{i+1}+\left|\frac{\partial v}{\partial r}\right|_{i+1} \\
k_{2} & =\left|E_{z}^{(j)}-E_{z}^{(j-1)}\right|_{i+1}+\left|E_{r}^{(j)}-E_{r}^{(j-1)}\right|_{i+1} \\
k_{2}^{\prime} & =\left|\frac{\partial E_{z}}{\partial u}\right|_{i+1}+\left|\frac{\partial E_{r}}{\partial v}\right|_{i+1} \\
C_{1} & =g(|z|+|r|) \\
C_{2} & =g(|u|+|v|) \\
g & =\text { maximum relative error }
\end{aligned}
$$

Before calculating the ultimate values $z_{i+1}, r_{i+1}$, $u_{i+1}, v_{i+1}$, the interval $\mathrm{h}$ is checked against the stability criterion (19). If satisfied, the next trajectory point is calculated with the same $h$. If not, $h$ is divided by two until criterion (19) is satisfied. Starting with $z_{i}$, $r_{i}, u_{i}, v_{i}$ seven points are then calculated with the Runge-Kutta method using the new $h$, and the solution continued.

If the stability criterion (19) can be satisfied with an interval twice as large, $h$ is doubled.and the next point with index $i+1$ is calculated from the points of index $i, i-2, i-4$, and $i-6$, instead of the usual $i$, $i-1, i-2$, and $i-3$.

\subsection{Interpolation of Potentials}

Potentials between mesh points are calculated by Lagrange interpolation. Let $n$ be the degree of interpolation. Then

$$
f(x)=\sum_{k=0}^{n} C_{k}(x) f\left(x_{k}\right)
$$

where

$$
C_{k}(x)=\frac{\left(x-x_{0}\right)\left(x-x_{1}\right) \ldots\left(x-x_{k-1}\right)\left(x-x_{k+1}\right) \ldots\left(x-x_{n}\right)}{\left(x_{k}-x_{0}\right)\left(x_{k}-x_{1}\right) \ldots\left(x_{k}-x_{k-1}\right)\left(x_{k}-x_{k+1}\right) \ldots\left(x_{k}-x_{n}\right)}
$$

For the mesh we have used, the absciscae are uniformly spaced with unit interval. Putting $u=x-x_{0}$ we have

$$
C_{k}(x)=\frac{u(u-1) \ldots(u-k+1)(u-k-1) \ldots(u-n)}{(-1)^{n-k} k !(n-k) !} .
$$


For our problem, the most reliable results were obtained with interpolation of order 4 .

The potential at point $P(r, z)$ is calculated as follows: (1) The potential at each point indicated in figure 3 with $x$ and 0 is interpolated from the potentials in the corresponding column or row. (2) Two values of the potential at $P$ are then interpolated, one from the potentials at points labeled $x$ and one from those labeled 0 . The average of these two values is taken as the potential at $P$.

\subsection{Calculation of Electric Field}

To find the two components of electric field, $E_{z}$ and $E_{r}$, at any point, we differentiate the Lagrange interpolating polynomials [eqs (25) and (26)] with respect to $z$ and $r$ respectively. There results:

$C_{k}^{\prime}(x)$

$$
\frac{\sum_{i=0 ; i \neq k}^{n} u(u-1) \ldots(u-i+1)(u-i-1) \ldots(u-k+1)(u-k-1) \ldots(u-n)}{(-1)^{n-k} k !(n-k) !}
$$

Here $x$ represents either $z$ or $r$ as appropriate. Again $n=4$ was found to give the smoothest results and was used in the program.

\subsection{Accuracy of Results}

It is difficult to predict, a priori, the accuracies of the potentials and trajectories obtained from our program. The accuracy of the relaxation technique, using five-point formulas, is usually taken to be of order $1 / N$ where $N$ is the number of mesh points. Since we used the more accurate nine-point formulas we believe that the accuracy of the potentials is considerably better than $10^{-4}$ of the maximum potential.

In the trajectory calculations the parameter $g$ in eq (24) gives the minimum precision with which each point of the trajectory is calculated. Focal lengths obtained from paraxial trajectories calculated with $g=10^{-5}$ satisfy the relationship

$$
\frac{f_{1}}{f_{2}}=\sqrt{\frac{V_{1}}{V_{2}}}
$$

to a precision of at least $10^{-5}$, demonstrating that the estimate of precision is realistic.

Even higher precision can be obtained by reducing $g$ with a consequent increase in computer time. The use of $g=10^{-5}$ is sufficient to determine accurate first-order focal properties and third-order aberration coefficients for the two-tube lens. Results will be reported in separate papers [9].

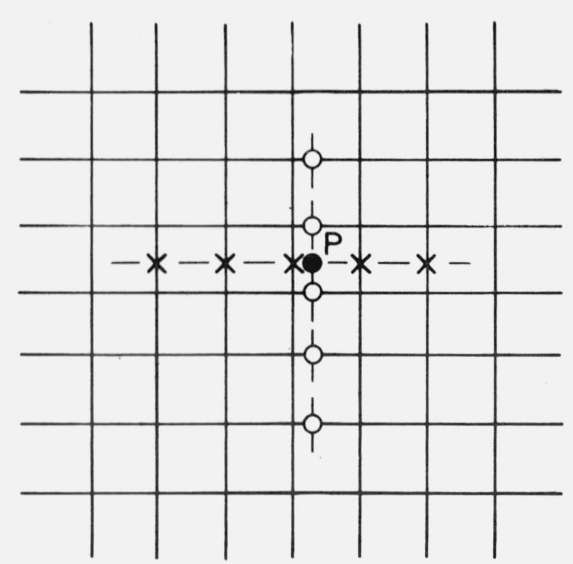

Figure 3. Points ( $\mathrm{x}$ and 0$)$ at which potentials are interpolated to calculate the potential at an arbitrary point $\mathrm{P}(\mathrm{r}, \mathrm{z})$.

\section{References}

[1] Boers, J. E., Digital computer analysis of axially symmetric electron guns, IEEE Trans. on Electron Devices 12, 425 (1965); Kirstein, P. T., and Hornsby, J. S., An Investigation into the use of iteration methods for the analysis of axially symmetric and sheet beam electrode shapes with an emitting surface, ibid 11, 196 (1964).

[2] Hildebrand, F. B., Introduction to Numerical Analysis (McGrawHill, New York, 1956). For a convenient tabulation of numerical differentiation formulas, see McCormick, J. M., and Salvadori, M. G., Numerical Methods in FORTRAN, p. 40 (Prentice-Hall, Englewood Cliffs, New Jersey, 1964).

[3] Carré, B. A., The determination of the optimum accelerating factor for successive over-relaxation, Computer J. 4, 73 (1963).

[4] Verster, J. L., On the use of gauzes in electron optics, Philips Res. Rpts. 18, 465 (1963).

[5] El-Kareh, A. B., Design and analysis of symmetrical and asymmetrical electrostatic immersion lenses using a digital computer, Record of 10th Symposium on Electron, Ion, and Laser Beam Technology, p. 393 (Ed. L. Marton, San Francisco Press, 1969); El-Kareh, A. B., and El-Kareh, J. C. J., Electron Beams, Lenses, and Optics, Vol. I (Academic Press, New York, 1970).

[6] Grivet, P., Electron Optics, p. 164 (Pergamon Press, Oxford, 1965).

[7] Read, F. H., Adams, A., and Soto-Montiel, J. R., Electrostatic cylinder lenses. I. Two element lenses, J. Phys. E (Sci. Instr.) 4, 625 (1971).

[8] Ralston, A., A First Course in Numerical Analysis (McGrawHill, New York, 1965).

[9] Natali, S., Di Chio, D., Uva, E., and Kuyatt, C. E., Accurate calculations of properties of the two-tube electrostatic lens. II. First order properties and P-Q curves. III. Aberration coefficients, submitted to Rev. Sci. Instr.; Kuyatt, C. E., Natali, S., and Di Chio, D., Third-order aberration coefficients of electron lenses, Proc. 11th Symp. Electron, Ion, and Laser Beam Technology, 1971, in press.

(Paper 76A1-695) 